\title{
DIE BRÜCKENMETAPHER IM POLITISCHEN SPRACHGEBRAUCH DER BUNDESKANZLERIN
}

Metaphern strukturieren unser Alltagsleben, also nicht nur unsere Sprache, sondern auch unser Denken und Handeln (Lakoff, Johnson 2011: 11). Aufgrund ihrer Eigenschaft des „highlighting and shading“ (ebd.: 18) sind Metaphern besonders gut geeignet, eine bestimmte Sichtweise auf einen Sach- oder Problemverhalt durchzusetzen und andere Aspekte zu verdunkeln. In diesem Beitrag soll am Beispiel der BRÜCKEN-Metapher im Sprachgebrauch der Bundeskanzlerin Angela Merkel gezeigt werden, wie sie versucht, „die Adressaten zu Komplizen ihrer Sichtweise zu machen“ (Strauß et al. 1989: 664). Dabei wird zunächst auf die verschiedenen Verwendungsweisen der BRÜCKEN-Metapher eingegangen sowie deren Bedeutungspotential rekonstruiert. Im zweiten Schritt wird veranschaulicht, welche sprachlichen Strategien die Kanzlerin mit dieser Metapher verfolgt. Im dritten Schritt wird gezeigt, wie mit Metaphern semantische Kämpfe ausgetragen werden. Den theoretischen und methodologischen Rahmen liefern die Konzeptuelle Metapherntheorie und die Politolinguistik.

Schlüsselwörter: Politolinguistik, Metaphern, Brückenmetaphorik, semantische Kämpfe

\section{Einleitung}

\subsection{Untersuchungsgegenstand}

Metaphern sind ein bewährter und unentbehrlicher Bestandteil des politischen Diskurses. Wegen ihrer persuasiven und handlungsanweisenden Kraft werden Metaphern von Politikern bewusst

jasmina12mahmutovic@hotmail.com 
in Anspruch genommen. In diesem Beitrag werden BRÜCKEN-Metaphern, die im politischen Sprachgebrauch der Bundekanzlerin Angela Merkel omnipräsent sind, aus sprachlichen Belegen herausgefiltert und analysiert. Von den zahlreichen Facetten, die bei der Metaphernanalyse mitbedacht werden können, interessieren in diesem Beitrag (1) die persuasive Leitungskraft von Metaphern und (2) die mit Metaphern ausgetragenen semantischen Kämpfe. Ziel und Erkenntnisinteresse ist es aufzuzeigen, wie positiv-evaluierte BRÜCKEN-Metaphern das kollektive Bewusstsein persuasiv lenken, indem sie die öffentliche Akzeptanz der verfolgten Politik stützen und die Notwendigkeit der ergriffenen Maßnahmen legitimieren (Lakoff, Johnson 2011: 180), (Lüger 2002: 457). Das Korpus, aus dem die nachfolgend analysierten Belege stammen, besteht aus Pressemitteilungen und Reden der Bundeskanzlerin, die auf ihrer Webseite www.bundeskanzlerin.de veröffentlicht wurden. Das Material wurde ergänzt um Pressetexte aus den Publikationen Der Spiegel, Focus, Handelsblatt und Wirtschaftswoche.

\subsection{Theoretischer und methodischer Rahmen}

Theoretisch und methodisch bewegt sich dieser Beitrag innerhalb des Forschungsparadigmas der Konzeptuellen Metapherntheorie (Lakoff, Johnson 2011) und der Politolinguistik. Die Politolinguistik beschäftigt sich inhaltlich mit der öffentlich-politischen Kommunikation (Girnth 2002: 1). Dem sprachkonstruktivistischen Ansatz folgend, geht sie davon aus: [...] daß sich Politik in Sprache vollzieht, daß politische Tätigkeit sprachliche Tätigkeit ist (Heringer 1990:9).

Politik wird verstanden als „die Kunst in Medium der Öffentlichkeit Zustimmungsbereitschaft zu erzeugen" (Lübbe 1975: 87). Sprechverwendung in der Politik ist interessengeleitet und appellativ, es geht um das Überreden (gefühlsmäßig-rhetorisch) und das Überzeugen (argumentativ-rational) (Burkhardt 2008). Politisches Handeln setzt bestimmte persuasive Fähigkeiten des Politikers voraus, denn „Politiker appellieren an die Emotionen ihres Publikums, 
sie buhlen um seine Zustimmung und sie werben für Ihre Überzeugungen (Girnth 2002: 1“).

Gerade bei gesellschaftlich relevanten und brisanten Themenkomplexen versuchen Politiker durch die Auswahl spezifischer sprachlicher Mittel ihre eigene Sichtweise als die einzig richtige durchzusetzen und die Deutungshoheit über umstrittene Begriffe zu gewinnen. Solche Versuche, „bestimmte sprachliche Formen als Ausdruck spezifischer, interessensgeleiteter und handlungsleitender Denkmuster durchzusetzen" (Felder 2006: 13) werden in der Politolinguistik als „semantischer Kampf“ bezeichnet. Wenn Gegner den Eindruck haben, ein Ausdruck werde allzu sehr mit den inhaltlichen Vorstellungen des politischen Gegners verknüpft, dann bleibt ihnen nur übrig, ihnen den „semantischen Fehdehandschuh“ (Klein 1989: 21) zuzuwerfen. Semantische Kämpfe werden mit unterschiedlichen sprachlichen Mitteln ausgetragen. Auch Metaphern gehören zum politischen Kampf um die Sprache (Skirl, Schwarz 2007: 76). Metaphern haben grundsätzlich etwas Ambivalentes: einerseits können sie politische und wirtschaftliche Prozesse, „die niemand richtig versteht" (Lakoff, Johnson 2011: 45), transparenter und leichter verständlich machen; andererseits werden sie häufig als Mittel eingesetzt, komplizierte Zusammenhänge entscheidend zu verkürzen und die Adressaten persuasiv zu lenken. Gerade konventionelle Metaphern, wie die hier besprochene BRÜCKEN-Metapher, sind im Denken und im Sprechen derart eingeschliffen, dass sie von den Adressaten als selbstverständlich angenommen und nicht mehr hinterfragt werden. Wenn von Politikern bestimmte Metaphern gezielt verwendet werden, kommt es zu polemischen Einseitigkeiten. Als probates Mittel gegen solche Metaphern kann mit Lakoff und Johnson ein bewusstes Dagegensetzen alternativer Metaphern empfohlen werden. Solche Metaphern können unsere „Erfahrung umgestalten“ (Jäkel 2003: 35) und ganz neue Einsichten hervorbringen.

Die hier analysierte BRÜCKEN-Metapher gilt als häufig verwendetes Metaphernkonzept im Bereich des öffentlich-politischen Sprechens. Als Basiskonzept (Schwarz, Chur 2007) eignet sich die BRÜCKEN-Metapher besonders gut, um abstrakte politische Sach- 
verhalte bildlich vor Augen zu führen. Konzepte der Basisebene entspringen der natürlichen Erfahrung und weisen eine lange Merkmalliste auf. Durch den metaphorischen Rückgriff auf ein solches Konzept werden abstrakte, vage strukturierte Konzepte kognitiv erfasst (Lakoff, Johnson 2011: 135).

Das Konzept BRÜCKE weist folgende Bedeutungsaspekte auf: Stabilität und Statik, Sicherheit und Schutz, Verbindung zweier getrennter Bereiche, Grenze und Übergang, Gefahr und Bewährung, Zwischenraum und Zusammenbruch, Moderne und Fortschritt (Metzler 2012: 63-64). Eine Brücke ist nur sinnvoll da, wo es etwas zu überspannen gibt: einen Fluss, eine Schlucht, einen Abgrund, zwei getrennte Bereiche, die sie miteinander verbindet. Als Symbol der Verbindung zweier getrennter Bereiche verknüpft die Brücke Gegensätze und fungiert als Bindeglied zwischen dem Bekannten und dem Unbekannten. Diese positive Deontik, im Sinne Hermanns (2012), macht sie zu einem Allzweckmittel in der politischen Kommunikation.

Im empirischen Teil dieses Beitrags wird zunächst auf die verschiedenen Verwendungsweisen der BRÜCKEN-Metapher im Sprachgebrauch der Bundeskanzlerin eingegangen sowie deren Bedeutungspotential rekonstruiert. Im zweiten Schritt soll veranschaulicht werden, welche sprachlichen Strategien die Kanzlerin mit dieser Metapher verfolgt. Im dritten Schritt soll gezeigt werden, wie ihre politischen Gegner versuchen, diese Metapher umzuwerten und unbrauchbar zu machen.

Zur systematischen Erfassung und Rekonstruktion der kognitiven Metaphern wird in diesem Beitrag der lexikalische Ansatz angewendet (Lakoff 1993: 244). Bei diesem Analyseverfahren wird von den konkreten sprachlichen Ausdrücken ausgegangen und schrittweise hin zu den abstrakten, kognitiven Metaphern geschlossen.

\section{Empirischer Teil}

Die Auswertung des Textkorpus zeigte, dass die Bundeskanzlerin die BRÜCKEN-Metapher in unterschiedlichen Zusammenhängen 
verwendet und dabei unterschiedliche Bedeutungsaspekte hervorhebt. Die im Korpus aufgefundenen sprachlichen Metaphern aus dem Ausgangsbereich BRÜCKE wurden identifiziert und nach Metaphernbereichen klassifiziert. Besprochen und interpretiert werden nur die prägnanten Konzeptualisierungen AusLÄNDISCHE MitBÜRGer Als BrüCKe, Versöhnung als BrüCKEnBAU und GeldPolitiSCHE MASSNAHMEN ALS BRÜCKE. Diese erste, grobe Klassifikation ermöglicht die erste Einsicht, dass es sich bei den metaphorisierten Konzepten - ausländische Mitbürger, Versöhnung und geldpolitische Maßnahmen - um brisante Sachverhalte handelt. Ein Thema ist brisant, wenn sich eine große Anzahl von Menschen von diesem Thema betroffen fühlt und den Diskurs darüber mit entsprechend großer emotionaler Beteiligung verfolgt bzw. aktiv an ihm teilnimmt (Felbick 2003: 33-34).

\subsection{Ausländische Mitbürger als Brücke}

Einwanderung ist ein gesellschaftspolitisch brisanter Themenbereich und zieht sich wie ein roter Faden durch die Geschichte der Bundesrepublik. Die Einstellungen zu Migranten unterscheiden sich diametral. Befürworter der Einwanderung unterstreichen die Notwendigkeit der Einwanderer für die deutsche Wirtschaft, während Gegner eine fremdenabwehrende Haltung einnehmen. Im Folgenden soll nun gezeigt werden, welche Sichtweise die Bundeskanzlerin den Adressaten über die Metapher AusLÄNDISchE MITBÜRGER ALS BRÜCKE vermittelt. Diese Metapher lässt sich aus folgenden sprachlichen Belegen rekonstruieren:

(1) Als Brücke zwischen den Ländern wirkten die 370.000 kroatischen Staatsbürger, die in Deutschland leben [...] Das ist, wie es die Bundeskanzlerin gesagt hat, nicht nur eine patriotische Brücke, sondern auch eine wirtschaftliche Brücke. (18.05.2019)

(2) Die 400.000 Serben, die in Deutschland leben, seien wie eine Brücke, die beide Länder miteinander verbinde. [...] „Deutschland und Serbien haben einen Warenaustausch von vier Milliarden Euro." (27.02.2018) 
(3) Die Menschen türkischer Abstammung in Deutschland sind eine wirkliche Brücke für ein enges Verhältnis beider Länder. Deutschland ist der wichtigste Handelspartner der Türkei. (04. 02. 2014)

(4) Dabei zählt unsere wirtschaftliche Kooperation zweifellos zu den wichtigsten Pfeilern der breiten Brücke, die unsere beiden Länder verbindet. (28.06.2011)

Zur Konzeptualisierung von ausländischen Mitbürgern beutet die Kanzlerin die positiven Bedeutungsaspekte des Konzepts BRÜCKE aus: BRÜCKE als Symbol der Verbindung zweier getrennter Bereiche und als Bindeglied zwischen dem Eigenen und dem Fremden. Durch die Wahl der BRÜCKEN-Metapher wird eine bestimmte Sichtweise auf Menschen mit Migrationshintergrund nahegelegt: als Vermittler zwischen Deutschland und ihrem jeweiligen Herkunftsland. Dadurch wird im Kontext der Politik ein positives Menschenbild entworfen. Das Wunschprofil wird weiter ausgebaut mit Ausdrücken aus dem Frame WIRTSCHAFT: wirtschaftliche Brücke (Beleg 1), Warenaustausch (Beleg 2), Handelspartner (Beleg 3), wirtschaftliche Kooperation (Beleg 4). Durch Einbettung in den Frame WIRTSCHAFT werden ausländische Mitbürger positiv ins rechte Licht rückt: weil sie uns helfen, unsere wirtschaftlichen Beziehungen mit ihrem jeweiligen Herkunftsland zu intensivieren, sind sie für Deutschland keine Belastung, sondern eine nützliche Bereicherung. Mit dem Frame WIRTSCHAFT lässt es sich gut argumentieren, sind doch alle Bürger in wirtschaftliche Abläufe eingebunden. Diese Einbindung ist unausweichlich und überlebensnotwendig. Der semantische Mehrwert der Metapher AusläNdische MitbürgER ALS BRÜCKE liegt darin, dass sie gängige Einstellungen modifiziert. Mit ihr wird die klischeehafte Metaphorisierung von Migranten ALS GEFÄHRLICHE WASSERMASSE (Strom, Flut, Welle) konzeptuell und emotional umgedeutet. Durch den semantischen Kampf des Umdeutens (Klein 1991: 57) wird die negative Deontik der WASSERMASSEN-Metapher mit dem positiven Glanz der BRÜCKEN-Metapher getilgt. 


\subsection{Versöhnung als Brückenbau}

In Gesellschaft und Politik wird Versöhnung als Bestandteil einer Vergangenheitsbewältigung und Konfliktregelung betrachtet. Versöhnung setzt voraus, dass man Schuld auf sich geladen hat. Durch die Metaphorisierung von Versöhnung als Brückenbau signalisiert die Bundeskanzlerin Handlungswille und Bereitschaft zur Wiedergutmachung.

(5) Man sprach von einer „Erbfeindschaft“ zwischen Deutschland und Frankreich. Das alles galt es zu überwinden und zu sagen: Schluss damit! Sich die Hände reichen! Brücken bauen! Wir wollen gerne Brückenbauer auch jenseits Europas sein. (30. Mai 2014)

(6) Wir verdanken dies denjenigen, die nach dem Zivilisationsbruch der Shoa in unser Land zurückkehrten, um hier zu leben und am Neuanfang mitzuwirken - die ihre Hand zur Versöhnung ausstreckten und damit Brücken über Gräben bauten, die schier unüberwindbar schienen. (9. November 2016)

(7) Deutschland weiß um die Kraft der Versöhnung. Denn wir Deutsche durften sie nach den Abgründen der beiden Kriege des vergangenen Jahrhunderts erfahren. Frankreich hat Deutschland die Hand zur Versöhnung gereicht. Deutschland wird das nie vergessen. Deutschland hat diese Hand dankbar angenommen. (11. November 2009)

Die Brücke der Versöhnung ist nur sinnvoll da, wo es eine Kluft zu überwinden gilt. Diese Kluft wird in den zitierten Belegen explizit genannt: „Erbfeindschaft" zwischen Deutschland und Frankreich (5) der Zivilisationsbruch der Shoa (6), die beiden Kriege des vergangenen Jahrhunderts (7). Es handelt sich um emotional hochgeladene Begriffe. Mit der BRüCKEN-Metapher werden die „Bewusstseins-, Denk- und Gefühlsprozesse der Textrezipienten” (Schwarz-Friesel 2015: 143) maßgeblich beeinflusst und deren Blick in die Zukunft gelenkt. Traumatische Erfahrungen aus der eigenen Geschichte werden über das Konzept des BRÜCKENBAUS als überwindbar dargestellt. Bauen ist ein transitives Verb, genauer gesagt ein Verb 
des Schaffens (Duden 2016: 400), das einen dynamischen Prozess beschreibt. In seiner Aktionsart ist bauen durativ und in seiner Aktionalität ein Handlungsverb (ebd: 419). Mit dem Konzept des BRÜCKEnBaus wird der Prozess der Versöhnung, Aussöhnung und Annäherung als BAUPROZESs fokussiert, womit dynamische Aspekte hervorgehoben und Veränderungen konzeptualisiert werden. Die Menschen werden aufgefordert, an diesem Prozess teilzunehmen: Brücken bauen! Wir wollen gerne Brückenbauer auch jenseits Europas sein (Beleg 5). Suggeriert wird: so wie eine wirkliche Brücke auch die tiefsten Schluchten überbrücken kann, können die düsteren Kapitel in der europäischen und deutschen Geschichte durch ein konstruktives Aufeinanderzugehen überwunden werden.

\subsection{Geldpolitische Maßnahmen als Brücke}

Der Metaphernbereich GeLDPolitische Massnahmen ALs BRÜCKE lässt sich in zwei Metaphernkonzepte ausdifferenzieren, die im Folgenden dargestellt und nacheinander abgearbeitet werden.

\subsubsection{Hartz IV als Brücke}

Arbeit ist eins der Schlüsselwörter der deutschen Gesellschaft und Kultur (Hermanns 2012: 277). Die Angehörigen dieser Gesellschaft definieren sich und andere durch die Art und Menge ihrer Arbeit. Für die Achtung, die einem andere entgegenbringen, wie auch die Selbstachtung ganz entscheidend wichtig ist auch, ob man Arbeit hat oder arbeitslos ist. Arbeitslose sind in den Augen mancher Zeitgenossen ein „Versager", weil sie sich vom arbeitenden Teil der Gesellschaft finanzieren lassen. Nun soll anhand von sprachlichen Belegen zum Thema Hartz IV gezeigt werden, wie die Bundeskanzlerin mit diesem brisanten Thema umgeht.

(8) Hartz IV ist keine Lebensaufgabe, sondern eine Brücke durch eine schwierige Zeit. (03.12.2010)

(9) Wir haben allerdings auch gesagt: Hartz IV, Arbeitslosengeld II - das ist kein Lebenszustand, sondern das ist eine Brücke über 
eine schwierige Zeit, wobei alles getan werden muss, um Anreize zu schaffen, Arbeit wieder aufzunehmen. (23.11.2010)

(10) Aber sie können nur Brücken auf dem Weg zu dauerhaften Jobs sein. (04.12.2014)

Über das sprachliche Bild der BRÜCKE wird Arbeitslosengeld als Verbindungsstück zwischen Arbeitslosigkeit und dauerhaftem Job perspektiviert. Gleichzeitig wird die Kurzfristigkeit der Maßnahme betont. So wie man eine Brücke rasch überquert, so ist auch Hartz IV als vorübergehendes Arbeitslosengeld in Anspruch zu nehmen. Die BRÜCKEN-Metapher wird „spezifisch rezipientenbeeinflussend“ (Schwarz-Friesel 2015:144) eingesetzt, um Einstellungen zu modifizieren. Explizit kommt diese Bewertung in den Ausdrücken keine Lebensaufgabe (Beleg 8) und kein Lebenszustand (Beleg 9) zur Geltung.

\subsubsection{Krisenüberwindung als Brücke}

Die nachfolgenden Belege stammen aus einer Zeit, in der Deutschland von der Weltwirtschaftskrise ab 2008 erschüttert war. Es mussten milliardenschwere Pakete zur Rettung der Wirtschaft - auf Kosten der Steuerzahler, die nicht gefragt wurden - verabschiedet werden. Daher galt es, um die Zustimmung des Publikums zu buhlen und die Notwendigkeit der Maßnahmen zu legitimieren.

(11) Mit diesem Maßnahmenpaket bauen wir für Bürger wie Unternehmer eine Brücke für Investitionen und Sicherung von Beschäftigung, bis der Aufschwung wieder aus eigener Kraft greift. (04.11.2008)

(12) Im Zuge der Finanz- und Wirtschaftskrise war es erforderlich geworden, eine Brücke über das tiefe Tal der Konjunktur zu bauen, erinnert die Kanzlerin. Das sei mit der Kurzarbeit und den Investitionsprogrammen auch recht gut gelungen. (03.07.2010) 
(13) Wir hoffen - ich weiß, das ist das Prinzip Hoffnung, aber wir können nichts anderes machen, als Brücken zu bauen -, dass Ende dieses Jahres die Wachstumspfade international, von den asiatischen bis zu den amerikanischen Märkten, wieder ansteigen und wir dann auch auf einen vernünftigen Pfad kommen. (03.07.2009)

Über die positiv-konnotierte BRÜCKEN-Metapher wird die Krise als überwindbar dargestellt. Die BRÜCKEN-Metapher wirkt beruhigend. Es werden keine Ängste geschürt und auch keine Bedrohungsszenarien entworfen. Die Kanzlerin signalisiert somit Handlungswille, Zuversicht und Selbstbewusstsein. Das, was überbrückt werden soll, wird entweder sachlich-nüchtern mit dem Pseudoterminus Krise bzw. Finanz- und Wirtschaftskrise versprachlicht oder mit der positiv anmutenden LANDSCHAFTS-Metapher vernetzt. Mit den Ausdrücken das tiefe Tal der Konjunktur (Beleg 12), Wachstumspfad, vernünftigen Pfad (Beleg 13) wird das Bild einer malerischen LANDSCHAFT gezeichnet und ökonomische Entwicklungen als eine Art BERGSTEIGEN perspektiviert, bei dem es abwärts oder aufwärts gehen kann. Die LANDSCHAFTs-Metaphorik kennt keine Verursacher und demnach keine Schuldigen. Der Akzent liegt auf den Handlungen, die über die BRÜCKEN-Metapher als vorübergehende, dem Gemeinwohl dienende Maßnahme dargestellt wird. Unliebsame geldpolitische Maßnahmen werden über die Metaphorisierung als BRÜCKE als etwas für den Steuerzahler Nützliches und insgesamt Positives konstruiert. Nebenbei teilt die Bundeskanzlerin mit: In Zeiten der Wirtschaftskrise, die Unübersehbarkeit, Risiko und Unberechenbarkeit bedeutet, steht ihre Partei für wirtschaftliche und soziale Sicherheit und Verlässlichkeit. Deutlich wird das am dem Personalpronomen wir in den Belegen (11) und (13). Die BRÜCKEN-Metapher dient der zentralen Strategie der Selbstdarstellung und Selbstinszenierung.

Durch die Wahl der LANDSCHAFTs-Metaphorik zur Konzeptualisierung der Krise werden andere zur Verfügung stehende metaphorische Alternativen abgewählt, wie etwa TsunAmI oder ABgRund. So wurde in Deutschland zur Vorbereitung des sogenannten Bankenrettungspakets monatelang die KRISE als ABGRUND 
verklärt, den man mit viel Geld zuschütten müsse, und eine Dollarflut beschworen, die es einzudämmen gelte.

Beim Sprachgebrauch der Kanzlerin handelt es sich um eine persuasive Strategie der konzeptuellen und emotionalen Umdeutung der negativ-wertenden FLUT- UND ABGRUND-Metaphorik. Mit der positiven BRÜCKENBAU-Metapher wird die negative Deontik der FLUT und des ABGRUNDS aufgebrochen und diese Krisenmetaphern somit unbrauchbar gemacht. Die Bundeskanzlerin scheint sich der Bedrohlichkeit dieser Metaphern bewusst zu sein und meidet sie konsequent in den ersten Monaten der Krise. Erst nachdem die Maßnahmen zur Wirtschaftsrettung im Bundestag verabschiedet wurden, mutierte die Krise zum ABGRUND:

(14) Wir mussten Banken retten, weil sie die Welt durch exzessive Arbeit an den Rand des Abgrunds gebracht haben. (01.03.2010)

(15) Ich glaube, heute sind wir alle in Europa der Meinung, dass das unter dem Strich insgesamt der richtige Weg war, um Europa vor einem Abgrund zu bewahren. (02.11.2010)

(16) Doch es ist der Bundesregierung gelungen, international und national die richtigen Lehren aus der Krise zu ziehen. „Wir haben den Absturz in den Abgrund verhindert", sagte Merkel. Zugleich erinnerte Merkel daran, dass die Krise noch nicht vorbei ist. Es gehe jetzt darum, „klug aus dem Tal wieder herauszukommen“. (20.11.2010)

Mit der ABGRUND-Metapher wird die Notwendigkeit der ergriffenen Maßnahmen noch einmal legitimiert, indem aufgezeigt wird, was auf dem Spiel stand und verhindert werden konnte. Die Ausdrücke Banken retten (14), der richtige Weg (15), klug (16) dienen der Imagearbeit und der Selbstaufwertung.

\section{Zusammenfassung der Analyseergebnisse}

Im Folgenden sollen die inhaltlichen Ergebnisse, die die Analyse der BRÜCKEN-Metaphorik im Sprachgebrauch der Bundeskanz- 
lerin erbracht hat, resümiert und die eingangs aufgestellten Fragen beantwortet werden. Die auffallende Häufigkeit der BRÜCKEN-Metapher in der öffentlich-politischen Kommunikation der Bundeskanzlerin wurde zum Anlass genommen, um zwei Forschungsfragen nachzugehen. Zum einen sollte nachgezeichnet werden, welche Sehweisen, Bewertungen und Deutungen mit dieser Metapher nahegelegt werden. Zum anderen sollte veranschaulicht werden, welche sprachlichen Strategien die Kanzlerin mit dieser Metapher verfolgt.

Die Bundeskanzlerin verwendet die BRÜCKEN-Metapher im Zusammenhang mit umstrittenen Themenfeldern wie ausländische Mitbürger, Versöhnung und geldpolitische Maßnahmen, und bekundet somit eine positive Einstellung zu diesen Themenkomplexen. Die Verwendungsfrequenz der BRÜCKEN-Metapher ist als Hinweis darauf zu lesen, dass es sich nicht um einen Zufall, sondern um eine Strategie handelt. Durch Steigerung der Verwendungsfrequenz soll die Trefferwahrscheinlichkeit erhöht werden, gerade wenn unterschiedliche Einschätzungen bezüglich eines brisanten Themas vorliegen. Zudem verwendet die Bundeskanzlerin eine Strategie, die in der Politolinguistik als „Ausbeuten von Assoziationen“ bezeichnet wird (Klein 1991: 65). Diese Strategie besteht darin, die eigene Position mit positiv konnotierten Wörtern im Bewusstsein möglichst vieler Menschen zu verknüpfen, so dass diese Wörter auf die eigene Partei abfärben. Durch den häufigen Gebrauch der BRÜCKEN-Metapher inszeniert sich die Bundeskanzlerin als BRÜCKENBAUERIN und knüpft an den Topos des Pontifex maximus an. Die Kommunikation zwischen Mensch und Gott bedarf in der römisch-christlichen Tradition eines obersten Brückenbauers, des Pontifex maximus in der Person des Papstes. Diesen Topos macht sich die Bundeskanzlerin zunutze, um sich als Vermittlerin zwischen dem abstrakten Gebilde Staat und den Bürgern zu inszenieren. Dass diese Strategie tatsächlich greift, wird durch den Umstand bestätigt, dass die Kanzlerin durch die Medien hinweg als Brückenbauerin bezeichnet wird.

(17) Angela Merkel. Die Brückenbauerin (Spiegel, 20.11.2006)

(18) Brückenbauerin Merkel (Handelsblatt, 27.05.2010) 
Zusammenfassend ist festzuhalten, dass die Bundeskanzlerin die BRÜCKEN-Metapher für sich und ihre eigene Partei reklamiert, um zentrale politische Positionen und Werte zu markieren.

Nachdem die ersten zwei Forschungsfrage beantwortet wurde, soll im nächsten Kapitel auf die dritte Forschungsfrage eingegangen werden. Es wird gezeigt, wie ein Gegner der Bundeskanzlerin versucht, die BRÜCKEN-Metapher mithilfe eines semantischen Tricks umzuwerten und für sie unbrauchbar zu machen.

\section{Der semantische Kampf um Merkels BRÜCKEN-Metapher}

Die BRÜCKEN-Metapher fungiert im Sprachgebrauch der Kanzlerin vorrangig als „Fahnenwort” d.h., „daß an ihm Freund und Feind den Parteistandpunkt, für den sie stehen, erkennen sollen“ (Hermanns 1982: 91). Im semantischen Kampf um die deontische Bedeutungskomponente von Fahnenwörtern bemühen sich die Gegner - um in der Metaphorik zu bleiben - die Fahne zu zerfetzen oder zu beschmutzen, jedenfalls ihr die Leuchtkraft zu nehmen (vgl. Klein 1991: 62). Im Folgenden soll gezeigt werden, wie der semantische Kampf um die BRÜCKEN-Metapher ausgetragen wird.

Im letzten direkten Schlagabtausch vor der Bundestagswahl 2013 hatte die Bundeskanzlerin die Sozialdemokraten in einer Sendung der ARD als „europapolitisch unzuverlässig“ charakterisiert. Ihr Gegenkandidat für das Kanzleramt, SPD-Kandidat Peer Steinbrück, reagiert empört und tritt zum semantischen Kampf an.

(19) Direkt an Merkel gewandt drohte er: „Sie müssen wissen, dass Sie damit Brücken zerstören." Die SPD habe die Euro-Rettungsmaßnahmen der Regierung im Bundestag bisher mitgetragen. (Spiegel 03.09.2019)

(20) Im letzten direkten Schlagabtausch vor der Wahl warf SPD-Kandidat Peer Steinbrück der CDU-Vorsitzenden am Dienstag im Bundestag vor, auf diese Weise „Brücken zu zerstören“. (Focus 03.09.2019)

Steinbrück verwendet in diesen Belegen die Strategie des Umdeutens und des Umwertens (Klein 1989, 1991). Die BRÜCKEN-Me- 
tapher, die die Kanzlerin für ihre Partei semantisch besetzt hat, wird um Bedeutungsaspekte aus dem toten Winkel der Metapher ergänzt. Über das Verb mitgetragen (Beleg 19) bringt Steinbrück seine Partei als PfEILER in die BRÜCKEN-Metapher der Kanzlerin ein und leistet damit zweierlei: Erstens wertet er das „exklusive wir" der Kanzlerin in ein „inklusives wir" um. Zweitens signalisiert er: die SPD ist ein wichtiger und unentbehrlicher Teil von Merkels politischem Erfolg. Zusätzlich interpretiert Steinbrück die BRÜCKEN-Metapher vorsorglich negativ, indem er ihr negative Bedeutungsaspekte hinzufügt. Mit der Äußerung Brücken zerstören rüttelt er an den Grundfesten der BRÜCKEN-Metapher und führt vor Augen, dass seine Zusammenarbeit mit der Bundeskanzlerin wie eine BRÜCKE zerstörbar ist und einstürzen kann. Mit einem weiteren semantischen Trick versucht er das Image der Bundeskanzlerin als BRÜCKENBAUERIN zu zerstören. Über den Vorwurf, metaphorische BRÜCKEN zu zerstören, erscheint sie nun in einem ganz anderen Licht: als SABOTEURIN. Die Medien greifen diesen semantischen Schlagabtausch auf und verpacken ihn in eine Schlagzeile:

(21) Merkel zerstört „Brücke“ zu Steinbrück (Handelsblatt 03.09.2013)

(22) Merkel vs. Steinbrück

„Sie zerschlagen Brücken“ (Spiegel 03.09.2013)

Die Sprachspiel mit Brücke und Steinbrück, die Umlaute und ähnlichen Wörter erinnern an die Textsorte „Zungenbrecher“. Mithilfe dieser Intertextualisierung wird Belustigung signalisiert und eine weitere Zusammenarbeit zwischen Merkel und Steinbrück als schier unmöglich bewertet.

\section{Fazit}

Das primäre Forschungsziel bestand darin, anhand der BRÜCKEN-Metapher im Sprachgebrauch der Kanzlerin aufzuzeigen, wie Metaphern aufgrund ihres Fokussierungseffekts auch Anlass für öf- 
fentlich ausgetragene semantische Kämpfe bieten. Es konnte gezeigt werden, dass die BRÜCKEN-Metapher unterschiedliche Funktionen in den analysierten Texten erfüllt. Erstens werden mit dieser Metapher umstrittene Themenfelder wie ausländische Mitbürger, Versöhnung und geldpolitische Maßnahmen nicht nur kognitiv erfasst, sondern auch in einem positiven Licht dargestellt. Somit wird die Brisanz dieser Themenfelder vorsorglich abgemildert. Zweitens wurde deutlich, dass die Kanzlerin die BRÜCKEN-Metapher im semantischen Kampf um die Sprache verwendet, um andere negativ-konnotierte Metaphern aus dem politischen Diskurs zu verdrängen. Drittens wurde veranschaulicht, dass die positiven Assoziationen der BRÜCKEN-Metapher durch den stetigen und konsequenten Gebrauch auf die Bundeskanzlerin abfärben und diese folglich in der Öffentlichkeit als BRÜCKENBAUERIN wahrgenommen wird. Und viertens wurde vor Augen geführt, wie ihr politischer Gegner versucht, die BRÜCKEN-Metapher ins Negative umzudeuten und somit unbrauchbar zu machen.

\section{LITERATURVERZEICHNIS}

Burkhardt 2008: Burkhardt, A. (2008). Deutsche Sprachgeschichte und politische Geschichte, in: Handbücher zur Sprach- und Kommunikationswissenschaft 2 / 1. Sprachgeschichte 2. Aufl., Berlin / New York: de Gruyter, 98 - 122.

Duden 2016: Duden Grammatik 9. Aufl. Berlin: Dudenverlag.

Felbick 2003: Felbick, D. (2003). Schlagwörter der Nachkriegszeit 1945 1949. Berlin, New York: de Gruyter.

Felder 2006: Felder, E. (2006): Semantische Kämpfe in Wissensdomänen. Eine Einführung in Benennungs-, Bedeutungsund Sachverhaltsfixierungs-Konkurrenzen. In: E. Felder (Hg.), Semantische Kämpfe. Macht und Sprache in den Wissenschaften, Berlin/New York: de Gruyter, 13-46.

Girnth 2002: Girnth, H. (2002). Sprache und Sprachverwendung in der Politik. Eine Einführung in die linguistische Analyse öffentlichpolitischer Kommunikation. Tübingen: Max Niemeyer.

Heringer 1990: Heringer H. J. (1990). Ich gebe Ihnen mein Ehrenwort: Politik, Sprache, Moral. München: Beck. 
Hermanns 2012: Hermanns, F. (2012): Wortgeschichtliche Analysen zur Zeitgeschichte, in

H. Kämper, A. Linke, M. Wengeler (Hg.), Der Sitz der Sprache im Leben. Beiträge zu einer kulturanalytischen Linguistik, Berlin, Boston: de Gruyter, 277 - 359.

Jäkel 2003: Jäkel, O. (2003). Wie Metaphern Wissen schaffen: die kognitive Metapherntheorie und ihre Anwendung in Modell-Analysen der Diskursbereiche Geistestätigkeit, Wirtschaft, Wissenschaft und Religion. Hamburg: Dr. Kovač.

Klein 1989: Klein, J. (1989). Politische Semantik. Bedeutungsanalytische und sprachkritische Beiträge zur Politischen Sprachverwendung. Opladen: Westdeutscher Verlag.

Klein 1991: Klein, J. (1991). Kann man 'Begriffe besetzen`? Zur linguistischen Differenzierung einer plakativen politischen Metapher, in F. Liedtke, M. Wengeler, K. Böke, (Hg.): Begriffe besetzen. Strategien des Sprachgebrauchs in der Politik. Opladen: Westdeutscher Verlag, 44-69.

Lakoff 1993: Lakoff, G. (1993): The Contemporary Theory of Metaphor, in A. Ortony, Metaphor and Thought, Cambridge: Cambridge University Press, 202 - 251.

Lakoff, Johnson 2011: Lakoff G., Johnson M. (2011). Leben in Metaphern. Konstruktion und Gebrauch von Sprachbildern. Heidelberg: Carl Auer. Lübbe 1975: Lübbe, H. (1975), Der Streit um Worte. Sprache und Politik, in: Kaltenbrunner, Gerd-Klaus (Hg.): Sprache und Herrschaft. Die umfunktionierten Wörter, Freiburg: Herder, 87 - 111.

Lüger 2002: Lüger, H. H. (2002), Berichten und Argumentieren im Konflikt. Akzeptanzwerbung, Desinformation und „rhetorischer Overkill“, in I. Pohl, Semantische Aspekte öffentlicher Kommunikation, Frankfurt am Main: Peter Lang, 439 - 468.

Metzler 2011: Metzler Lexikon literarischer Symbole. Stuttgart, Weimar: J. B. Metzler.

Schwarz-Friesel 2015: Schwarz-Friesel, M. (2015), Metaphern und ihr persuasives Inferenzpotenzial. Konzeptualisierungen des islamistischen Terrorismus nach 9/11 im massenmedialen Diskurs, in C. Spieß, Metapher und Metonymie (Hg.), Berlin/New York: de Gruyter, 144 - 160.

Schwarz, Chur 2007: Schwarz, M., Chur, J. (2007): Semantik. Ein Arbeitsbuch. Tübingen: Gunter Narr.

Skirl, Schwarz 2007: Skirl, H., Schwarz, M. (2007): Metapher. Heidelberg: Universitätsverlag Winter. 
Strauß et al. 1989: Strauß, G., Haß, U., Harras, G. (1989). Brisante Wörter von Agitation bis Zeitgeist. Ein Lexikon zum Sprachgebrauch. Berlin/ New York: de Gruyter.

\title{
Jasmina Mahmutović
}

\section{METAFORA MOSTA U POLITIČKOJ KOMUNIKACIJI KANCELARKE ANGELE MERKEL}

\begin{abstract}
Rezime
Autorica u ovom radu analiza i interpretira jezičke instance pojavljivanja pojmovne metafore MOST u medijskim članicima i političkim govorima njemačke kancelarke Angele Merkel. Polezeći od teze da metafore imaju moć uvjeravanja, što ih čini omiljenim jezičkim sredstvom političkog djelovanja i semantičke borbe (Klein 1991, Felder 2006), nastoji se pokazati da se ova metafora u javnopolitičkoj komunikaciji koristi ciljano i svjesno u svrhu iskazivanja vlastitog i odbacivanja tuđeg stava. Analiza je rađena metodama kognitivne semantike na korpusu od 22 članka objavljena na web stranici kancelarkewww.bundeskanzlerin.de, odnosno uštampanim medijima Der Spiegel, Focus, Handelsblatt und Wirtschaftswoche. $\mathrm{Na}$ osnovu empirijske analize dolazi se do zaključka da pojmovna metafora MOST ispunjava nekoliko različitih funkcija: (1) funkciju ublažavanja kontroverznih društveno-političkih tema: stranci, suočavanje sa prošlošću i potrošnja javnih sredstava; (2) funkciju jačanja vlastitog stava i potiskivanja tuđih stavova iz diskursa i (3) funkciju jačanja imidža same kancelarke i njenje političke stranke. $\mathrm{Na}$ zadnjem nivou analize ilustrira se jezički fenomen semantičke borbe na primjeru metafore MOST.
\end{abstract}

Ključne riječi: politolingvistika, kognitivna teorija metafore, pojmovna metafora MosT, semantička borba 